\title{
Line versus representational bisections in unilateral spatial neglect
}

\author{
S Ishiai, Y Koyama, K Seki, M Izawa
}

\begin{abstract}
Objectives-To clarify the mechanisms of left unilateral spatial neglect found in the bisection of lines after cueing to the left end point and to determine whether neglect occurs for the mental representation of a line.

Methods-A new representational bisection task was developed to eliminate the influence of the right segment of the physical line that would attract attention. Eight patients with typical left unilateral spatial neglect underwent line and representational bisection tasks on a computer display with a touch panel. In the line bisection with cueing, they bisected a line after touching the left end point. In the representational bisection, the patients were presented with a line until they touched the left end point. On the blank display, they pointed to the subjective midpoint of the erased line. The performances of the two bisection tasks were compared when the length and position of stimulus lines were varied.
\end{abstract}

Results-The rightward errors in the representational bisection were greater than or equivalent to those in the line bisection with cueing. The effect of line length in which the errors became greater for the longer lines was equally found in the line bisection with cueing and the representational bisection. This was confirmed in the condition where the right end point was placed at a fixed position and the line length was varied.

Conclusions-After cueing to the left end point, rightward bisection errors of patients with neglect are not caused by overattention to the right segment of the physical line. Left neglect occurs mainly for the mental representation formed at the time of cueing or seeing the whole extent of a line.

(F Neurol Neurosurg Psychiatry 2000;69:745-750)

Keywords: unilateral spatial neglect; line bisection; representation; attention

Patients with left unilateral spatial neglect bisect horizontal lines with rightward errors. ${ }^{1}$ Left neglect is often reduced but remains apparent even after they are cued to the left end point when the whole extent is projected onto the attentive right visual field. ${ }^{2}{ }^{3}$ As we reported in a previous study, ${ }^{4}$ their gaze tends to return quickly from the left end point to the point where the subjective midpoint is later placed. Although the left part of the line falls in the inattentive or defective left field of vision after cueing, its mental representation may be stored. By contrast, the right part continues to be perceived in the right visual field. When bisecting a line after cueing, patients with neglect seem to bisect the extent that consists of the mental representation on the left side and the real line segment on the right side.

Patients with left neglect sometimes show representational neglect when they describe the left side of internally generated images. ${ }^{56}$ For external visual stimuli, Bisiach et al and Ogden et at asked patients with neglect to detect differences within pairs of patterns moving leftward or rightward behind a narrow vertical slit. The detection of the mentally reconstructed images was deficient on the left side. Rightward errors in the line bisection after cueing to the left end point may result from left neglect for the mental representation of a line. On the other hand, presence of objects in the non-neglected right space worsens leftward exploration. ${ }^{910}$ In the clock drawing, the left side hours may be transposed to the right side where the numbers were previously drawn. ${ }^{11}$ Patients with typical neglect often exhibit magnetic gaze attraction towards the right side. ${ }^{12}$ Accordingly, it is possible that rightward errors of bisection with cueing results from overattention to the line segment seen in the right visual field and impaired disengagement of attention from that part.

The present study aimed to clarify the mechanisms of neglect found in the bisection of lines after cueing to the left end point. We investigated the effect of the line segment perceived in the right visual field on line bisection performance and attempted to determine whether left neglect occurs for the mental representation of a line. We devised a representational bisection task using a computer display with a touch panel. Patients with neglect were presented with a line until they were cued to touch its left end point and saw the whole extent. They then pointed to the subjective midpoint on the blank display. Their performance in the representational bisection was compared with that in the line bisection with cueing. If left neglect occurs for the mental representation formed at the time of cueing to the left end point, rightward errors should be seen in either line or representational bisection. However, the amount of errors might decrease in the representational bisection where there is no visual stimulus that would attract attention to the right side.

\section{Subjects and methods}

SUBJECTS

Eight right handed patients with typical left unilateral spatial neglect after a right hemispheric infarction were studied. They showed 
Table 1 Patients' clinical data

\begin{tabular}{|c|c|c|c|c|c|c|c|c|}
\hline \multirow[b]{2}{*}{ Patient } & \multirow[b]{2}{*}{ Age (y) } & \multirow[b]{2}{*}{ Sex } & \multirow[b]{2}{*}{$\begin{array}{l}\text { Duration } \\
\text { after onset } \\
\text { (months) }\end{array}$} & \multirow[b]{2}{*}{$\begin{array}{l}\text { Visual } \\
\text { field }\end{array}$} & \multicolumn{3}{|c|}{ Unilateral spatial neglect } & \multirow[b]{2}{*}{ Lesion } \\
\hline & & & & & $\begin{array}{l}\text { Line } \\
\text { bisection }\end{array}$ & Copying & $\begin{array}{l}\text { Line } \\
\text { cancellation }\end{array}$ & \\
\hline 1 & 73 & $\mathrm{~F}$ & 1.5 & VE & + & +++ & + & FTP \\
\hline 2 & 76 & $\mathrm{~F}$ & 2 & IQ & ++ & +++ & +++ & $\mathrm{ftPo}$ \\
\hline 3 & 64 & M & 3.5 & IQ & +++ & + & ++ & fTPo \\
\hline 4 & 70 & $\mathrm{~F}$ & 7 & VE & ++ & ++ & ++ & FTPo \\
\hline 5 & 68 & $M$ & 3 & $\mathrm{H}$ & ++ & +++ & ++ & $\mathrm{tP}$ \\
\hline 6 & 48 & M & 5 & $\mathrm{H}$ & +++ & + & + & ftp \\
\hline 7 & 73 & $\mathrm{~F}$ & 1 & IQ & + & +++ & ++ & $\mathrm{ftPo}$ \\
\hline 8 & 69 & M & 9 & IQ & +++ & + & + & dfp \\
\hline
\end{tabular}

$+=$ Mild; $++=$ moderate; $+++=$ severe; VE=visual extinction; IQ=inferior quadrantanopia; $\mathrm{H}=$ hemianopia; $\mathrm{F}$ or $\mathrm{f}=$ large or small frontal lesion; $\mathrm{T}$ or $\mathrm{t}=$ large or small temporal lesion; $\mathrm{P}$ or $\mathrm{p}=$ large or small parietal lesion; $\mathrm{o}=$ small occipital lesion; $\mathrm{d}=$ deep lesion.

left neglect in the line cancellation test, ${ }^{13}{ }^{14}$ the copying of a daisy, ${ }^{15} 16$ and the line bisection test. $^{115}$ Their neglect in the three tests was rated as mild, moderate, or severe according to the scales of Levine et $a l^{17}$ and Ishiai et $a .^{15}$ Brain MRI disclosed that they had an infarction that involved the right parietal lobe mainly and extended into one or more of the frontal, temporal, and occipital lobes. Four patients had left inferior quadrantanopia and two had left homonymous hemianopia on confrontation testing. The remaining two patients showed left sided visual extinction on double simultaneous stimulation. The mean age of the patients was 67.6 (SD 8.7). Table 1 presents the patients' clinical data. Ten age matched healthy subjects (mean age 66.2 (SD 6.8)) served as normal controls. All patients and control subjects gave informed consent to participate in this study.

\section{APPARATUS}

Line or representational bisection tasks were performed on a 12 inch liquid crystal display (LCD) monitor with a touch panel (Gunze, AV7624FT). The resolution was set at $600 \times 800$ pixels. The monitor was placed in front of the subjects so that its centre was positioned in the sagittal midplane of the trunk. Head movement was not restricted. The viewing distance was about $400 \mathrm{~mm}$. The subjects responded to stimuli by touching the translucent surface panel with a pencil-like pointer. A personal computer (IBM, ThinkPad 310) controlled the presentation of stimuli and recorded the responses.

LINE AND REPRESENTATIONAL BISECTION TASKS A horizontal black line $2 \mathrm{~mm}$ wide was presented against the white background at the level of the centre of the monitor. The subjects pointed to the subjective midpoint with the pointer held in the right hand. Before starting each bisection trial, the hand was always placed in the subjects' natural and comfortable position on their knees.

Line bisection without cueing

A line was displayed on the monitor $1000 \mathrm{~ms}$ after a short alarm sound. The subjects bisected the line by pointing to the subjective midpoint. The computer recorded its location to the closest $\mathrm{mm}$ and produced a short vertical mark $10 \mathrm{~mm}$ tall and $1 \mathrm{~mm}$ wide at that point.
Line bisection with cueing

A line was presented in the same way as in the line bisection without cueing. The subjects were first asked to touch the left end point of the line with the pointer. When they touched the left end point accurately, the computer emitted a short sound. If they failed to do so, the trial was restarted after the examiner's encouragement to find the left end point. After the cueing, they pointed to the subjective midpoint and the computer recorded its location, where a short vertical mark appeared.

\section{Representational bisection}

The subjects were presented with a line in the same way as in the other bisection tasks and asked to point to its left end point. When they touched the left end point accurately, the computer generated a short sound and the line disappeared. On the blank display, they pointed to the subjective midpoint of the erased line. The computer recorded the location of the subjective midpoint and produced a mark there.

\section{Position and length of lines}

In the centre condition, a line 100,150 , or 200 $\mathrm{mm}$ long was displayed centrally on the LCD monitor. In the right condition, a 100,150 , or $200 \mathrm{~mm}$ line was presented so that its right end point was always positioned $100 \mathrm{~mm}$ to the right of the centre of the monitor.

\section{Procedure}

All patients and control subjects were examined with the three bisection tasks in the centre condition in one session that took about 30 minutes. They first underwent the line bisection without cueing. The line bisection with cueing and the representational bisection were then given to them. They received two or three practice trials before starting experimental bisections of each task. The order of the line bisection with cueing and the representational bisection was couterbalanced with an ABBA design. In each bisection task, they performed 10 experimental trials for each length in randomised order completing a total of 30 bisections.

Four patients (3, 4, 6, and 7) and all control subjects were further examined in the right condition. The arrangement of the three tasks was the same as in the centre condition. This session was given to the patients on the day after the centre condition. The controls completed the centre condition and then the right condition with a pause of 5 minutes.

BISECTION JUDGMENT IN THE RIGHT VISUAL FIELD

Two patients ( 3 and 4) were asked to tell the longer segment of a line transected by a short vertical mark while they kept pointing to the left end point. A 100, 150, or $200 \mathrm{~mm}$ line with a mark was displayed horizontally across the centre of the monitor. The deviation of the mark from the true centre ranged from $-25 \%$ (leftward) to $+25 \%$ (rightward) of the total length in $5 \%$ increments. The mark thus appeared at one of the 11 different positions, in which the true centre was included. As in the 
Table 2 Mean deviations in bisection tasks

\begin{tabular}{|c|c|c|c|c|c|c|}
\hline & \multicolumn{3}{|l|}{ Control subjects } & \multicolumn{3}{|c|}{ Patients with neglect } \\
\hline & $100 \mathrm{~mm}$ & $150 \mathrm{~mm}$ & $200 \mathrm{~mm}$ & $100 \mathrm{~mm}$ & $150 \mathrm{~mm}$ & $200 \mathrm{~mm}$ \\
\hline \multicolumn{7}{|l|}{ Centre condition: } \\
\hline LB without cueing & $-0.08(0.87)$ & $0.26(1.73)$ & $0.36(1.77)$ & $20.9(11.2)$ & $36.4(14.8)$ & $49.7(17.6)$ \\
\hline LB with cueing & $0.43(1.12)$ & $0.50(1.42)$ & $0.30(2.28)$ & $4.5(3.6)$ & $10.6(6.0)$ & $18.4(7.0)$ \\
\hline Representational bisection & $0.66(3.36)$ & $0.83(4.16)$ & $0.78(3.43)$ & $12.2(8.2)$ & $19.1(9.6)$ & $25.7(10.9)$ \\
\hline \multicolumn{7}{|l|}{ Right condition: } \\
\hline LB without cueing & $0.21(1.02)$ & $1.11(2.01)$ & $0.89(2.51)$ & $3.9(17.6)$ & $25.8(18.2)$ & $50.7(16.9)$ \\
\hline LB with cueing & $0.61(1.59)$ & $0.81(2.08)$ & $0.93(2.26)$ & $6.6(6.1)$ & $17.0(10.4)$ & $32.5(13.7)$ \\
\hline Representational bisection & $0.64(2.55)$ & $1.59(3.99)$ & $2.41(3.84)$ & $10.6(10.7)$ & $22.7(12.1)$ & $39.5(15.5)$ \\
\hline
\end{tabular}

$\mathrm{LB}=$ line bisection

Values are means $(\mathrm{SD})$ in $\mathrm{mm}$.

landmark task of Harvey et $a l,{ }^{18}$ the patients were falsely informed that no line was correctly bisected. Five trials were performed at each

- Line bisection without cueing

$\Delta$ Line bisection with cueing

$\triangle$ Representational bisection

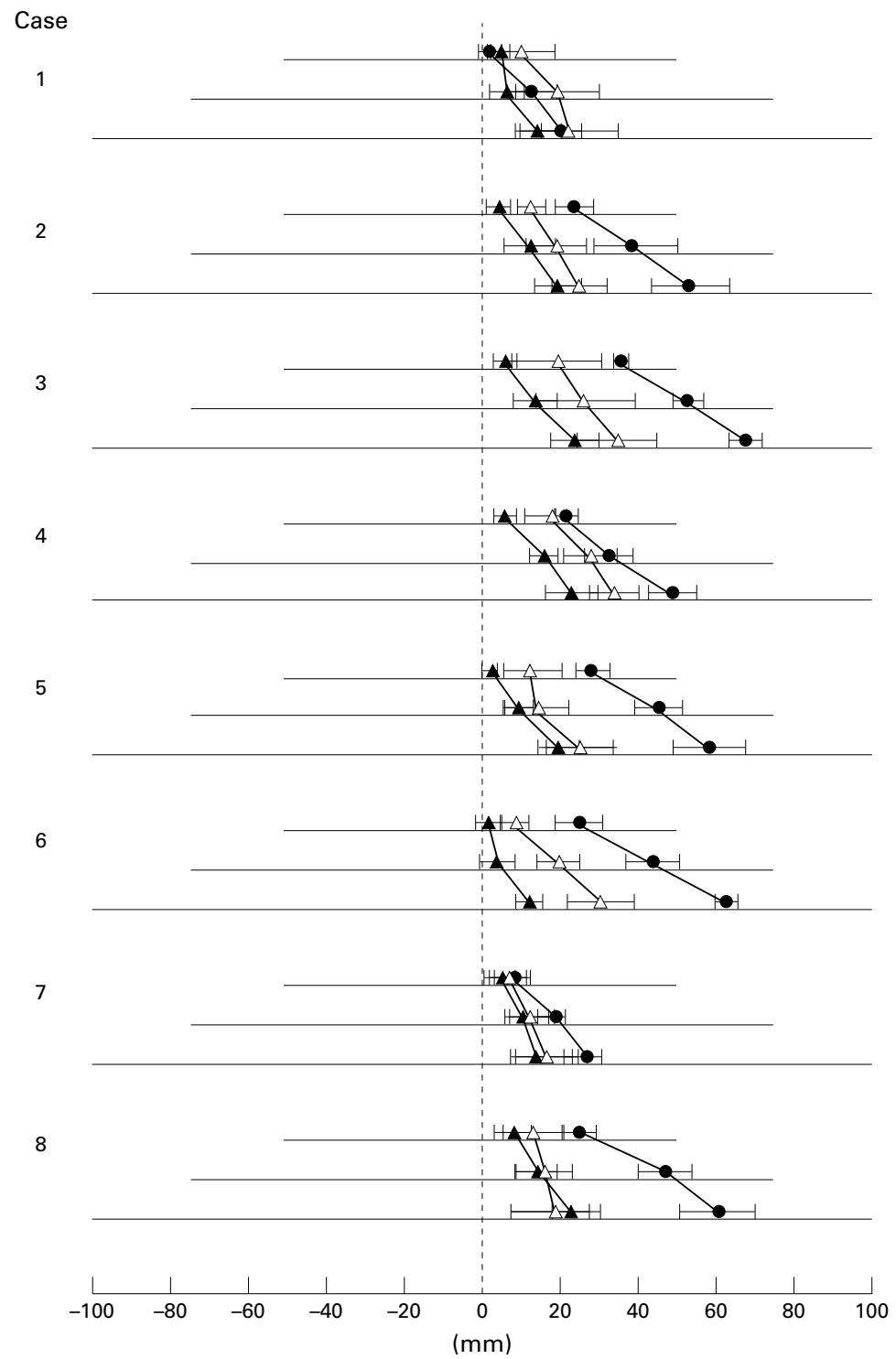

Figure 1 Bisection errors in the centre condition for individual patients with left unilateral spatial neglect. The mean locations (SD) of the subjective midpoint are shown separately for the 100, 150, and $200 \mathrm{~mm}$ lines. The horizontal axis indicates rightward deviations from the true centre. Closed circles=line bisection without cueing, black triangles=line bisection with cueing, white triangles=representational bisection. position of the mark for each length. The order of presentation was randomised across the positions of the mark and the line lengths.

\section{Results}

LINE AND REPRESENTATIONAL BISECTION TASKS Centre condition

Deviations of the subjective midpoint from the true centre were measured to the closest $\mathrm{mm}$, and rightward deviations were given positive values. Table 2 presents the mean deviations for the control subjects and the patients with unilateral spatial neglect. The control subjects marked the centre of the lines almost accurately in the line bisection without cueing, the line bisection with cueing, and the representational bisection. As shown in figure 1, all patients presented greater mean deviations than the control subjects in either task except for the $100 \mathrm{~mm}$ lines in the line bisection with cueing.

Deviations of the subjective midpoint were analysed with a two way analysis of variance (ANOVA) separately for individual patients. Firstly, we compared the results between the line bisection without cueing and the line bisection with cueing. The main factors were task and line length. The statistical results are summarised in table 3. For all patients, both main effects were significant: cueing to the left end point reduced rightward deviations and greater deviations were found for the longer lines. The length effect was smaller in the line bisection with cueing than in the line bisection without cueing, which explained the significant interaction between task and length. Secondly, the results were compared between the line bisection with cueing and the representational bisection (table 3 ). Six patients (1 to 6) showed greater mean deviations in the representational bisection than in the line bisection. The remaining two patients (7 and 8) showed no significant difference of deviation between the two bisection tasks. The effect of line length was significant in either task for all patients.

In the line bisection with cueing and the representational bisection, we analysed the latency from the time of touching the left end point to the time of pointing to the subjective midpoint. All patients showed no significant difference of latency between the two bisection tasks.

\section{Right condition}

Table 4 and figure 2 show the results of the bisection tasks for the four patients examined in the right condition. In the line bisection 
Table 3 Statistical results for bisection performances in centre condition

\begin{tabular}{|c|c|c|c|c|c|c|}
\hline \multirow[b]{2}{*}{ Patient } & \multicolumn{3}{|c|}{$L B$ without cueing $v L B$ with cueing } & \multicolumn{3}{|c|}{$L B$ with cueing $v$ representational bisection } \\
\hline & Task & Length* & Task $\times$ Length & Task & Length $^{\star}$ & Task $\times$ Length \\
\hline 1 & $\mathrm{p}<0.025$ & $\begin{array}{l}\mathrm{p}<0.001 \\
(\mathrm{p}<0.01 / \mathrm{p}<0.01)\end{array}$ & $\mathrm{p}<0.005$ & $\mathrm{p}<0.001$ & $\mathrm{p}<0.005$ & NS \\
\hline 2 & $\mathrm{p}<0.001$ & $\begin{array}{l}\mathrm{p}<0.001 \\
(\mathrm{p}<0.01 / \mathrm{p}<0.01)\end{array}$ & $\mathrm{p}<0.025$ & $\mathrm{p}<0.001$ & $\mathrm{p}<0.001$ & NS \\
\hline 3 & $\mathrm{p}<0.001$ & $\begin{array}{l}\mathrm{p}<0.001 \\
(\mathrm{p}<0.01 / \mathrm{p}<0.01)\end{array}$ & $\mathrm{p}<0.001$ & $\mathrm{p}<0.001$ & $\mathrm{p}<0.001$ & NS \\
\hline 4 & $\mathrm{p}<0.001$ & $\begin{array}{l}\mathrm{p}<0.001 \\
(\mathrm{p}<0.01 / \mathrm{p}<0.01)\end{array}$ & $\mathrm{p}<0.005$ & $\mathrm{p}<0.001$ & $\mathrm{p}<0.001$ & NS \\
\hline 5 & $\mathrm{p}<0.001$ & $\begin{array}{l}\mathrm{p}<0.001 \\
(\mathrm{p}<0.01 / \mathrm{p}<0.01)\end{array}$ & $\mathrm{p}<0.005$ & $\mathrm{p}<0.001$ & $\mathrm{p}<0.001$ & NS \\
\hline 6 & $\mathrm{p}<0.001$ & $\begin{array}{l}\mathrm{p}<0.001 \\
(\mathrm{p}<0.01 / \mathrm{p}<0.01)\end{array}$ & $\mathrm{p}<0.001$ & $\mathrm{p}<0.001$ & $\begin{array}{l}\mathrm{p}<0.001 \\
(\mathrm{p}<0.01 / \mathrm{p}<0.01)\end{array}$ & $\mathrm{p}<0.005$ \\
\hline 7 & $\mathrm{p}<0.001$ & $\begin{array}{l}\mathrm{p}<0.001 \\
(\mathrm{p}<0.01 / \mathrm{p}<0.01)\end{array}$ & $\mathrm{p}<0.005$ & NS & $\mathrm{p}<0.001$ & NS \\
\hline 8 & $\mathrm{p}<0.001$ & $\begin{array}{l}\mathrm{p}<0.001 \\
(\mathrm{p}<0.01 / \mathrm{p}<0.01)\end{array}$ & $\mathrm{p}<0.001$ & NS & $\mathrm{p}<0.001$ & NS \\
\hline
\end{tabular}

$\mathrm{LB}=$ line bisection.

$\star$ The effect of length in each task is shown in parentheses.

without cueing, patients 3, 4, and 6 showed greater mean deviations than the control subjects. The remaining patient (7) bisected the $200 \mathrm{~mm}$ lines with rightward deviations. In the line bisection with cueing and the representational bisection, the four patients showed greater mean deviations than the control subjects for the 150 and $200 \mathrm{~mm}$ lines.

In the line bisection without cueing, each patient placed the subjective midpoint about the same distance to the left of the right end point irrespective of the length of the lines. The distance from the right end point to the subjective midpoint (right distance) was not significantly different among the three line lengths for patients 3, 6, and 7. Patient 7 bisected the 100 $\mathrm{mm}$ lines with leftward errors and the $150 \mathrm{~mm}$ lines within the normal range. These findings

- Line bisection without cueing

$\Delta$ Line bisection with cueing

$\triangle$ Representational bisection

\section{Case}

3

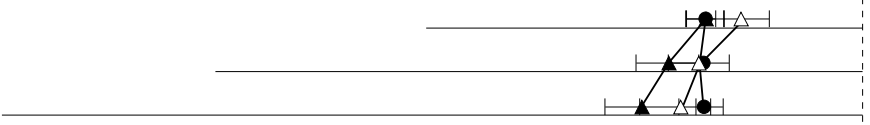

4

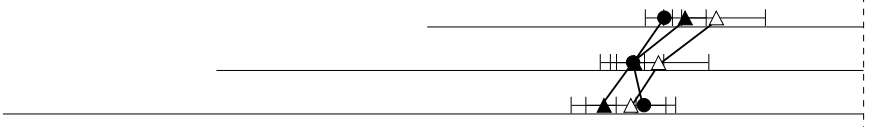

6

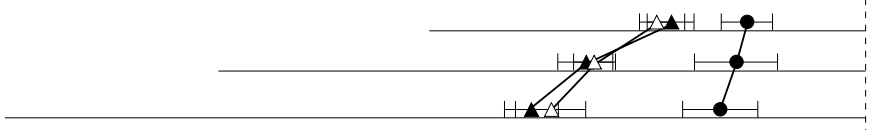

7

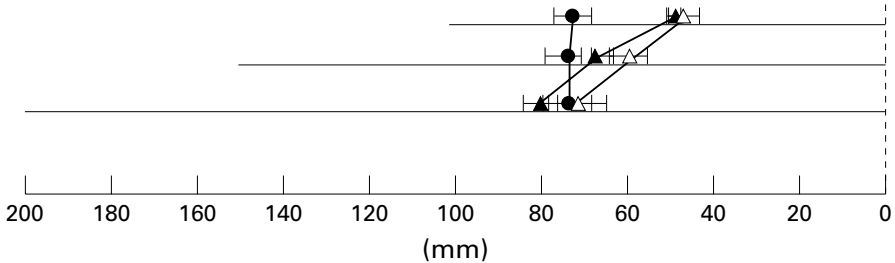

Figure 2 Bisection errors in the right condition for individual patients with left unilateral spatial neglect. The mean locations of the subjective midpoint (SD) are shown separately for the 100,150, and $200 \mathrm{~mm}$ lines. The horizontal axis indicates the distance from the right end point to the subjective midpoint. suggest that line length was hardly processed in their bisection without cueing. The right distance was therefore treated as a dependent variable for analysis of variance (ANOVA) to investigate how the length or the left end point of the lines was processed in the right condition. ${ }^{19}$

Data were submitted to a two way ANOVA with the same design as the centre condition. In the comparison between the line bisection without cueing and that with cueing (table 4), a significant interaction was found between task and length. For patients 3, 6, and 7, a significant effect of length was found in the line bisection with cueing but not in the line bisection without cueing. Patient 4 also showed a typical effect of length when cued to the left end point. Patient 7's leftward deviation for the $100 \mathrm{~mm}$ lines disappeared with cueing. Accordingly, the four patients showed neglect depending on line length when cued to the left end point. Reduction of rightward errors was apparent in only patient 6 .

The effect of length was similarly found in the line bisection with cueing and the representational bisection (table 4 ). Task $\times$ length interaction was not significant for patients 3,4 , and 6. Patients 3, 4, and 7 placed the subjective midpoint more rightward in the representational bisection than in the line bisection with cueing. Patient 6's bisection performance was not significantly different between the two tasks.

\section{BISECTION JUDGMENT IN THE RIGHT VISUAL} FIELD

When asked to keep pointing to the left end point, patient 3 said that the left segment was longer for all but one of the correctly bisected lines. Only one $200 \mathrm{~mm}$ line with a mark at its true centre was judged as having the longer right segment. Patient 4 judged the left segment as longer for all correctly bisected lines. Both patients always detected the longer right segment when presented with the lines that were bisected leftward by $5 \%$ of the total length. The other deviations of the mark were also appreciated without error. 
Table 4 Statistical results for bisection performances in right condition

\begin{tabular}{|c|c|c|c|c|c|c|}
\hline \multirow[b]{2}{*}{ Patie $n t$} & \multicolumn{3}{|c|}{$L B$ without cueing $v L B$ with cueing } & \multicolumn{3}{|c|}{$L B$ with cueing $v$ representational bisection } \\
\hline & Task & Length $^{\star}$ & Task $\times$ Length & Task & Length ${ }^{\star}$ & Task $\times$ Length \\
\hline 3 & $\mathrm{p}<0.001$ & $\begin{array}{l}\mathrm{p}<0.001 \\
(\mathrm{NS} / \mathrm{p}<0.01)\end{array}$ & $\mathrm{p}<0.005$ & $\mathrm{p}<0.001$ & $\mathrm{p}<0.001$ & NS \\
\hline 4 & NS & $\begin{array}{l}\mathrm{p}<0.001 \\
(\mathrm{p}<0.05 / \mathrm{p}<0.01)\end{array}$ & $\mathrm{p}<0.005$ & $\mathrm{p}<0.005$ & $\mathrm{p}<0.001$ & NS \\
\hline 6 & $\mathrm{p}<0.001$ & $\begin{array}{l}\mathrm{p}<0.001 \\
(\mathrm{NS} / \mathrm{p}<0.01)\end{array}$ & $\mathrm{p}<0.001$ & NS & $\mathrm{p}<0.001$ & NS \\
\hline 7 & $\mathrm{p}<0.001$ & $\begin{array}{l}\mathrm{p}<0.001 \\
(\mathrm{NS} / \mathrm{p}<0.01)\end{array}$ & $\mathrm{p}<0.001$ & $\mathrm{p}<0.001$ & $\begin{array}{l}\mathrm{p}<0.001 \\
(\mathrm{p}<0.01 / \mathrm{p}<0.01)\end{array}$ & $\mathrm{p}<0.05$ \\
\hline
\end{tabular}

$\mathrm{LB}=$ line bisection.

${ }^{\star}$ The effect of length in each task is shown in parentheses.

\section{Discussion}

The patients with left unilateral spatial neglect erred rightward when they bisected the mental representation of a line after seeing its whole extent in the right visual field. The rightward errors were greater than or equivalent to those in the line bisection with cueing to the left end point. In the representational bisection, the patients saw no line when marking the subjective midpoint. The results indicate that after cueing to the left end point, the presence of the right segment perceived in the right visual field had no effect on bisection performance of the patients with neglect.

Rightward errors from the true centre were greater for the longer lines in either the line bisection with cueing or the representational bisection. The right end point of the longer lines was located further to the right side in the condition where the lines were centred in the sagittal midplane of the trunk. In this centre condition, rightward errors would become greater for the longer lines even if only the location of the right end point was stored in memory. When patients with severe neglect bisect lines without cueing, they may determine the subjective midpoint according to the location of the right end point in the egocentric space. ${ }^{19}$ Our patients also seemed to bisect lines similarly in the right condition where the right end point was placed at a fixed position. They placed the subjective midpoint nearly the same distance to the left of the right end point irrespective of line length. With cueing to the left end point, however, the subjective midpoint was placed more leftward as the line extended more to the left side. This true effect of line length was equally found in the line bisection with cueing and the representational bisection. The results suggest that patients with neglect have a mental representation of a line that reflects the length of its whole extent that was projected onto the right visual field while cueing. We conclude that in the line bisection with cueing, neglect occurs mainly for the mental representation formed at the time of cueing or seeing the whole extent of a line. However, further study is necessary to determine whether neglect occurs for the accurate representation of a line or neglect results in forming the shorter representation.

Attention of patients with left unilateral spatial neglect may be shifted rightward also in the right visual field. ${ }^{20}{ }^{21}$ However, such bias of attention does not seem to cause rightward errors of line bisection after cueing. Our previ- ous studies ${ }^{45}$ disclosed that patients with neglect could appreciate their bisection errors when forced to fixate the left end point. Two patients in the present study were asked to judge the longer segment of lines with a transection mark while they kept pointing to the left end point. Their judgment was flawless when the deviation of the mark amounted to $5 \%$ or more of the line length. The lines with a mark at the true centre were almost always judged as having the longer left segment. This bias of bisection judgement corresponds well to the results of the recent study ${ }^{22}$ : normal subjects bisected a line slightly towards the central field when gaze was fixed so that the line was seen in only one hemifield. The patients with neglect should err leftward, if they bisected lines according to their judgement of the centre in the right visual field.

The patients often showed greater rightward errors in the representational bisection than in the line bisection after cueing to the left end point. Ishiai et $a l^{4}$ reported that the fixation of patients with left unilateral spatial neglect never returned to the left side after it departed from the cued left end point. In our patients, the latency from the time of cueing to the time of placing the mark was not different between the line bisection and the representational bisection. It is therefore unlikely that they traced the line leftward again after cueing and showed smaller errors in the line bisection. The presence of a real line may have affected the scanning from the left end point and reduced rightward errors of bisection.

Visual stimuli presented in the left visual field or the left space may be processed unconsciously. ${ }^{23}{ }^{24}$ Berti et al ${ }^{25}$ reported that the presence of irrelevant stimuli around the left part of a line improved bisection performance in some patients with neglect. They considered that implicit processing of the left sided stimuli oriented attention automatically to the left side. It is possible that after cueing to the left end point, our patients also paid attention covertly to the left extent of the line. In six of the eight patients, the left visual field was not completely defective. The left segment of the line may have fallen in the residual sector of the left visual field. The remaining two had left homonymous hemianopia but showed smaller rightward errors in the line bisection with cueing than in the representational bisection. Their hemianopia seen on confrontation testing might not be a true defect of visual field as reported in patients with severe neglect. ${ }^{26-28}$ 
The possibility remains that the frame of the display screen, especially the right edge, affected the representational bisections to increase rightward errors. If the patients used the right edge as a cue to judge the centre of the erased lines, the effect of line length on the bisection errors should become smaller compared with that in the bisection of the physical lines. However, this was not seen. It is therefore unlikely that the increase of rightward errors in the representational bisection resulted from the use of the computer display, which was not wide enough to directly exclude the possibility.

All patients in the present study had a lesion that involved the right parietal lobe. The lesion extension to the frontal lobe seemed to have no effect on the results of the line and representational bisections. Exploratory motor deficit may predominate in neglect after damage to the frontal lobe. ${ }^{29}$ Our cueing technique probably eliminated the influence of directional hypokinesia and hypometria, ${ }^{30}{ }^{31}$ because the patients moved the hand from the left extreme of a line to mark the subjective midpoint. Whereas some patients with neglect with a frontal lesion perform normally in the line bisection test, ${ }^{32}$ others exhibit small but apparent rightward errors. ${ }^{33}$ There are controversies as to whether neglect in an internally generated image occurs after parietal or frontal lesions. ${ }^{64}$ We consider that the approach using the line and representational bisection tasks will also contribute to exploration of mental representation in patients with frontal lobe neglect.

This work is supported by a Grant in Aid for Scientific Research (C) to Sumio Ishiai from the Ministry of Education, Science, Sports, and Culture, Japan.

1 Heilman KM, Watson RT, Valenstein E. Neglect and related disorders. In: Heilman KM, Valenstein E, eds. Clinical neuropsychology, 3rd

2 Heilman KM, Valenstein E. Mechanisms underlying hemispatial neglect. Ann Neurol 1979;5:166-70.

3 Riddoch MJ, Humphreys GW. The effect of cueing on unilateral neglect. Neuropsychologia 1983;21:589-99.

4 Ishiai S, Seki K, Koyama Y, et al. Effects of cueing on visuospatial processing in unilateral spatial neglect. $f$ Neurol 1995;242:367-73.

5 Bisiach E, Luzzatti C. Unilateral neglect of representational space. Cortex 1978;14:129-33.

6 Bartolomeo P, D'Erme P, Gainotti G. The relationship between visuospatial and representational neglect. Neurology 1994;44:1710-4.

7 Bisiach E, Luzzatti C, Perani D. Unilateral neglect, representational schema and consciousness. Brain 1979; 102:609-18.

8 Ogden JA. Contralesional neglect of constructed visual images in right and left brain-damaged patients. Neuropsychologia 1985;23:273-7.
9 Mark VW, Kooistra CA, Heilman KM. Hemispatial neglect affected by non-neglected stimuli. Neurology 1988;38: 1207-11.

10 Làdavas $\mathrm{E}$, Umiltà $\mathrm{C}$, Ziani $\mathrm{P}$, et al. The role of right side objects in left side neglect: a dissociation between perceptual and directional motor neglect. Neuropsychologia 1993;31:761-73.

11 Di Pellegrino G. Clock-drawing in a case of left visuo-spatial neglect: a deficit of disengagement? Neuropsychologia 1995; 33:353-8.

12 Gainotti G, D'Erme P, Bartolomeo P. Early orientation of attention toward the half space ipsilateral to the lesion in patients with unilateral brain damage. $\mathcal{F}$ Neurol Neurosurg Psychiatry 1991;54:1082-9.

3 Albert ML. A simple test of visual neglect. Neurology 1973; 23:658-64.

14 Ishiai S, Sugishita M, Odajima N, et al. Improvement of unilateral spatial neglect with numbering. Neurology 1990; 40:1395-8.

15 Ishiai S, Furukawa T, Tsukagoshi H. Visuospatial processes of line bisection and the mechanisms underlying unilateral patial neglect. Brain 1989;112:1485-502.

16 Seki K, Ishiai S. Diverse patterns of performance in copying and severity of unilateral spatial neglect. $\mathcal{F}$ Neurol 1996;243: $1-8$.

17 Levine DN, Warach JD, Benowitz L, et al. Left spatial neglect: effects of lesion size and premorbid brain atrophy on severity and recovery following right cerebral infarction. Neurology 1986;36:362-6.

18 Harvey M, Milner AD, Roberts RC. An investigation of hemispatial neglect using the landmark task. Brain Cogn 1995;27:59-78

19 Koyama Y, Ishiai S, Seki K, et al. Distinct processes in line bisection according to severity of left unilateral spatial neglect. Brain Cogn 1997;35:271-81.

20 De Renzi E, Gentilini M, Faglioni P, et al. Attentional shift towards the rightmost stimuli in patients with left visual neglect. Cortex 1989;25:231-7.

21 Làdavas E, Petronio A, Umiltà C. The deployment of visual attention in the intact field of hemineglect patients. Cortex 1990;26:307-17.

22 Nielsen KE, Intriligator J, Barton JJS. Spatial representation in the normal visual field: a study of hemifield line bisection. Neuropsychologia 1999;37:267-77.

23 Volpe BT, Ledoux JE, Gazzaniga MS. Information processing of visual stimuli in an 'extinguished' field. Nature 1979; 282:722-4.

24 Marshall JC, Halligan PW. Blindsight and insight in visuo-spatial neglect. Nature 1988;336:766-7.

25 Berti A, Maravita A, Frassinetti F, et al. Unilateral neglect can be affected by stimuli in the neglected field. Cortex 1995;31:331-43.

26 Kooistra CA, Heilman KM. Hemispatial visual inattention as hemianopia. Neurology 1989;39:1125-7.

ker R, Findlay JM, Young AW, et al. Disentangling neglect and hemianopia. Neuropsychologia 1991;29:101927.

28 Vallar G, Sandroni P, Rusconi ML, et al. Hemianopia, hemianesthesia, and spatial neglect: a study with evoked potentials. Neurology 1991;41:1918-22.

29 Mesulam M-M. Large-scale neurocognitive networks and distributed processing for attention, language, and memory. Ann Neurol 1990;28:597-613.

30 Coslett HB, Bowers D, Fitzpatrick E, et al. Directional hypokinesia and hemispatial inattention in neglect. Brain 1990;113:475-86.

31 Mattingley JB, Bradshaw JL, Phillips JG. Impairments of movement initiation and execution in unilateral neglect. Directional hypokinesia and bradykinesia. Brain 1992;115: 1849-74.

32 Binder J, Marshall R, Lazar R, et al. Distinct syndromes of hemineglect. Arch Neurol 1992;49:1187-94.

33 Ishiai S, Watabiki S, Lee E, et al. Preserved leftward movement in left unilateral spatial neglect due to frontal lesions. F Neurol Neurosurg Psychiatry 1994;57:1085-90.

34 Guariglia C, Padovani A, Pantano P, et al. Uniltaral neglect restricted to visual imagery. Nature 1993;364:235-7. 